\title{
Acute Septic Arthritis of the Knee Caused by Kingella kingae in a 5-Year-Old Cameroonian Boy
}

\begin{abstract}
Nawal El Houmami ${ }^{1 *}$, Dimitri Ceroni ${ }^{2}$, Karine Codjo Seignon ${ }^{1}$, Jean-Christophe Pons ${ }^{1}$, Cédric Lambert ${ }^{3}$, Guillaume André Durand ${ }^{1}$, Philippe Minodier ${ }^{4}$, Léopold Lamah ${ }^{5}$, Philippe Bidet ${ }^{6}$, Jacques Schrenzel ${ }^{7}$, Didier Raoult ${ }^{1}$ and Pierre-Edouard Fournier ${ }^{1}$

${ }^{1}$ Research Unit on Infectious and Emerging Tropical Diseases (URMITE), UM63, CNRS 7278, IRD 198, INSERM 1095, Aix-Marseille Université, IHU Méditerranée Infection, Marseille, France, ${ }^{2}$ Département de l'enfant et de l'adolescent, Hôpital des Enfants, Hôpitaux Universitaires de Genève (HUG), Geneva, Switzerland, ${ }^{3}$ Department of Pediatrics, Dracénie Hospital, Draguignan, France, ${ }^{4}$ Department of Pediatric Emergency Medicine, North Hospital, Aix-Marseille Université, Marseille, France, ${ }^{5}$ Department of Orthopedics and Traumatology, Donka University Hospital, University of Conakry Gamal Abdel Nasser, Conakry, Guinea, ${ }^{6}$ Laboratoire de Microbiologie, Hôpital Robert Debré, Assistance Publique - Hôpitaux de Paris, Université Paris Diderot, Sorbonne Paris Cité, INSERM, IAME, UMR 1137, Paris, France, ${ }^{7}$ Bacteriology and Genomic Research Laboratories, Geneva University Hospitals (HUG) and Geneva University, Geneva, Switzerland
\end{abstract}

\section{OPEN ACCESS}

Edited by:

Frederick Robert Carrick, University of Cambridge, United Kingdom

Reviewed by: John Bernard Ziegler, Sydney Children's Hospital, Australia Enrique Medina-Acosta, State University of Norte Fluminense, Brazi Christian T. K.-H. Stadtlander, Independent Researcher, United States

*Correspondence: Nawal El Houmami nawal.elho@gmail.com

Specialty section: This article was submitted to Child Health and Human Development, a section of the journal

Frontiers in Pediatrics

Received: 25 August 2017 Accepted: 13 October 2017 Published: 06 November 2017

Citation:

El Houmami N, Ceroni D, Codjo Seignon K, Pons J-C, Lambert C, Durand GA, Minodier $P$, Lamah L, Bidet P, Schrenzel J, Raoult D and Fournier P-E (2017) Acute Septic Arthritis of the Knee Caused by Kingella kingae in a 5-Year-Old Cameroonian Boy. Front. Pediatr. 5:230. doi: 10.3389/fped.2017.00230
Kingella kingae is an important cause of invasive infections in young children from Western countries. Although increasing reports indicate that this organism is the leading agent of bone and joint infections in early childhood, data on K. kingae infections from resource-limited settings are scarce, and none has yet been reported in Africa. We herein report the diagnostic and epidemiological investigations of the first case of $K$. kingae arthritis identified in a child from sub-Saharan Africa. A 5-year-old Cameroonian boy presented with a sudden painful limp which appeared in the course of a mild rhinopharyngitis. He lived in Cameroon where he had been vaccinated with BCG at birth and moved to France for holidays 4 days before consultation. There was no history of trauma and he did not have any underlying medical condition. Upon admission, he had a temperature of $36.7^{\circ} \mathrm{C}$, and clinical examination revealed right-sided knee tenderness and effusion that was confirmed by ultrasound imaging. Laboratory results showed a white blood cell count of 5,700 cells $/ \mathrm{mm}^{3}$, C-reactive protein level of $174 \mathrm{mg} / \mathrm{L}$, and platelet count of 495,000 cells $/ \mathrm{mm}^{3}$. He underwent an arthrocentesis and was immediately given intravenous amoxicillin-clavulanate. Conventional cultures from blood samples and synovial fluids were negative. Polymerase chain reaction (PCR) assay targeting the broad-range 16S rRNA gene and real-time quantitative PCR assays targeting Mycobacterium species were negative. Surprisingly, real-time PCR assays targeting the $c p n 60, r t \times A$, and $r t \times B$ genes of $K$. kingae were positive. Multicolor fluorescence in situ hybridization specific for K. kingae identified the presence of numerous coccobacilli located within the synovial fluid. Finally, multilocus sequence typing analysis performed on deoxyribonucleic acid directly extracted from joint fluid disclosed a novel $K$. kingae sequence-type complex. This case report demonstrates that $K$. kingae may be considered as a potential cause of septic arthritis in children living in sub-Saharan Africa, and hence the burden of $K$. kingae infection may be not limited to the Western countries. Further studies are required to determine the prevalence of $K$. kingae infection and carriage in Africa.

Keywords: Kingella kingae, pediatrics, arthritis, infectious, multilocus sequence typing, Africa South of the Sahara 


\section{BACKGROUND}

Kingella kingae is an emerging pathogen recognized as the primary etiology of bone and joint infections in young children from Western countries $(1,2)$. Asymptomatically harbored in the oropharynx of children aged 6-48 months, the prevalence of $K$. kingae oropharyngeal carriage ranges from 8 to $23 \%$ from studies carried out in Israel, Switzerland, and New Zealand (3-6). Because this Gram-negative bacterium is usually responsible for a mild to moderate inflammatory response, and its detection is notoriously difficult by conventional culture, diagnosis of $K$. kingae infection requires a high index of suspicion and the use of adequate detection methods such as real-time quantitative polymerase chain reaction (qPCR) assays $(6,7)$. These molecular diagnostic tools exhibit higher sensitivity compared with culture methods, shorten the time of detection from days to a few hours, and enable the identification of the organism among healthy carriers (4-6).

Large-scale epidemiological studies based on multilocus sequence typing (MLST) analysis of $K$. kingae showed that dominant clones belonging to sequence-type complexes 6 (STc-6), $-14,-23$, and -25 accounted for $72 \%$ of strains disseminated worldwide, mainly in the USA, Europe, and Israel, with ST-14 and ST-25 being positively associated with osteoarticular infections (8). To date, K. kingae infection and carriage have been studied in Israel, Europe, North and South America, Australia, New Zealand, and Japan $(5,8-10)$, but none have yet been reported in Africa. We herein report the diagnostic and epidemiological investigations of $K$. kingae arthritis in a young, previously healthy child from Cameroon, and we discuss the clinical implications of these findings.

\section{CASE PRESENTATION}

On 11 July 2016, a 5-year-old Cameroonian boy was admitted to the emergency department at the Dracénie Hospital in the region Provences-Alpes-Côte d'Azur, France, due to a painful limp that appeared in the morning. He lived in Cameroon where he had been vaccinated with BCG at birth, and moved to Southeastern France for holidays 4 days before consultation. A mild rhinopharyngitis had occurred the previous week, but as the symptoms were mild, no treatment had been undertaken. There was no history of trauma, and he did not have any underlying medical condition. Upon admission to hospital, the child had a temperature of $36.7^{\circ} \mathrm{C}$ and refused to walk. Clinical examination revealed right-sided knee tenderness and effusion. Neither skin rash nor oral ulcerations were noted. Laboratory results showed an elevated C-reactive protein (CRP) level at $174 \mathrm{mg} / \mathrm{L}$, with normal white blood cell count of 5,700 cells $/ \mathrm{mm}^{3}$ and platelet count of 495,000 cells $/ \mathrm{mm}^{3}$. Ultrasound imaging confirmed effusion of the right knee, whereas conventional radiograph showed no significant abnormality. The child underwent an arthrocentesis, and mildly opaque and yellowish liquid was extracted, suggesting

Abbreviations: CRP, C-reactive protein; DNA, deoxyribonucleic acid; MLST, multilocus sequence typing; PCR, polymerase chain reaction; ST, sequence type; STc, sequence-type complex. a septic arthritis of the right knee. Consequently, the child was immediately given intravenous amoxicillin-clavulanate $100 \mathrm{mg} / \mathrm{kg}$ three doses daily during 3 days.

\section{DESCRIPTION OF LABORATORY INVESTIGATIONS AND DIAGNOSTIC TESTS}

Because conventional cultures applied for Gram-positive, Gramnegative, mycobacterial species, and fungi from the joint fluid and blood samples were negative, joint specimens were sent in dry ice to the molecular diagnosis laboratory of the URMITE unit in Marseille, where bacterial deoxyribonucleic acid (DNA) was extracted directly from the joint fluid. Polymerase chain reaction (PCR) assay targeting the broad-range bacterial 16S rRNA gene (11) and qPCR assays targeting both Mycobacterium species and Mycobacterium tuberculosis complex (12) were negative. Given the age of the patient, $K$. kingae was also sought by using specific qPCR assay targeting the K. kingae cpn60 (groEL) gene (11). Surprisingly, this specific $K$. kingae assay was positive, as well as qPCR assays targeting the Kingella-specific $r t x A$ and $r t x B$ genes $(7,13)$, thus confirming the diagnosis of septic arthritis caused by K. kingae. The organism was also identified by multicolor fluorescence in situ hybridization specific for K. kingae (Figures S1 and S2 in Supplementary Material), which revealed the presence of large numbers of viable coccobacilli located within the synovial fluid (Figure 1). Cardiac investigations ruled out endocarditis. A switch to oral amoxicillin-clavulanate $100 \mathrm{mg} / \mathrm{kg}$ three doses daily was then undertaken on 15 July 2016 and was planned for a total duration of 2 weeks. Despite these recommendations, the treatment was continued for another 2 months in Cameroon. During the final follow-up 3 months postoperatively, clinical examination revealed a normal knee status with a normal range of motion.

Thereafter, MLST studies using a modified protocol specific for $K$. kingae was performed on bacterial DNA extracted directly from the joint fluid as previously described (14). Five alleles were unambiguously identified, namely, $a d k-2$, aroE-2, cpn60-2, zwf-13, and recA-2. Unexpectedly, 14 single nucleotide variants of the $a b c Z$ allele were identified from nucleotides 6-447 (Figure S3 in Supplementary Material; Table 1). To estimate the between-strain relatedness and define an MLST scheme for K. kingae, a different allele number was given to each distinct sequence within a locus, and a distinct sequence-type (ST) number was attributed to each distinct allele combination (15). $K$. kingae isolates were then grouped into ST-complexes (STcs) if they differed at no more than one locus from at least one other member of the group. Among the 70 STs of K. kingae that are documented in the multilocus sequence database (MLST) of the Institut Pasteur database (http://bigsdb.pasteur.fr/perl/ bigsdb/bigsdb.pl? db=pubmlst_kingella_seqdef_public\&page $=$ downloadProfiles\&scheme_id=1), ST-26, which belongs to the highly invasive STc-25, was the closest ST by sharing four alleles, namely, adk-2, cpn60-2, gdh/zwf-13, and recA-2 with the causative strains that were herein identified (Table 2). Although analysis of the combination produced by the five unambiguous 


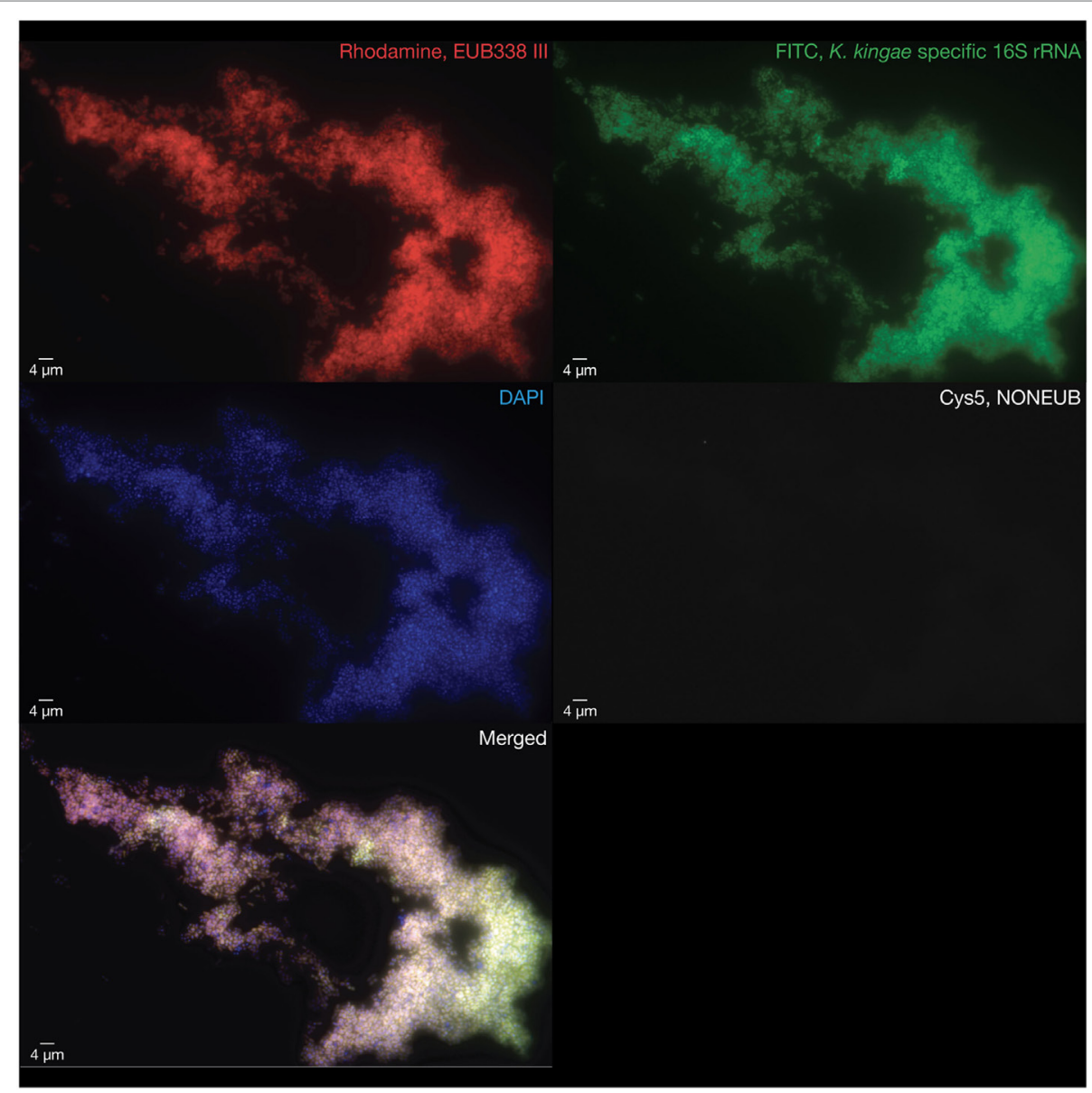

FIGURE 1 | Multicolor fluorescence in situ hybridization assays were performed on the pathogenic synovial fluid after this latter was formalin fixed and paraffin embedded. Large numbers of viable coccobacilli representing the causative Kingella kingae strains are visualized in red using a rhodamine-labeled probe targeting a consensus sequence of the bacterial 16S rRNA gene (EUB388 III, top left), in green using an FITC-labeled probe targeting the $K$. kingae-specific V1 region of the $16 S$ rRNA gene (top right), and in blue using a DAPI probe to label deoxyribonucleic acid (middle left). An internal negative control was performed by using a bacterial non EUB388 probe (middle right). The merged image was obtained summing the four abovementioned images (bottom left).

alleles indicated that the causative K. kingae strains belongs to a novel ST, the presence of multiple $a b c Z$ alleles does not allow to precisely define it. Moreover, in the MLST scheme of K. kingae, founder genotypes of STcs were defined as the ST of the STc with the highest number of neighboring STs [(15), Table 3]. Consequently, although analysis of the combination produced by the five unambiguous alleles indicated that the causative $K$. kingae strains belong also to a novel STc, no specific denomination is yet possible. Moreover, since each of these housekeeping genes is present in one copy in the whole genome of K. kingae, these findings suggested co-infection by strains belonging to distinct STs.

\section{DISCUSSION}

To the best of our knowledge, we herein report the first case of laboratory-confirmed invasive infection due to K. kingae in a child living in Africa. Little is known of the epidemiology of pediatric bone and joint infections in the African continent; however, it is largely recognized that Staphylococcus aureus is the most common pathogen cultured in children with septic arthritis in resource-limited settings $(10,16)$. Nevertheless, septic arthritis caused by $S$. aureus affects most frequently older children and is more prone to result in a higher systemic inflammatory response when compared with $K$. kingae infections, and the organism is recovered without difficulty by culture of blood and synovial fluid aspirates $(10,16,17)$. Although $K$. kingae arthritis is characterized by normal to moderate increase in inflammatory markers, we point out that the patient had a markedly elevated CRP level upon admission, consistent with invasive infection caused by K. kingae of at least several days duration. Despite this, K. kingae infection was highly suspected because this pathogen is recognized as the first cause of culture-negative, acute septic arthritis in young children and affects most commonly the knee (1). In addition, it was also demonstrated that viral respiratory infections may play a role in the pathogenesis of the disease by damaging the mucosal lining of the oral cavity, thus facilitating the spread of the organism from blood to distant anatomic sites (2). 
TABLE 1 | Distance matrices of the Kingella kingae abcZ allele corresponding to the MAFFT alignment displayed in Figure S3 in Supplementary Material.

\begin{tabular}{|c|c|c|c|c|c|c|c|c|c|c|c|c|c|c|c|c|c|c|c|c|c|c|}
\hline & 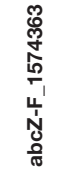 & 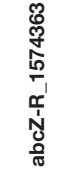 & 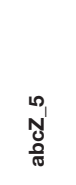 & $\begin{array}{l}\bar{N} \\
\text { U } \\
\text { त }\end{array}$ & $\begin{array}{l}N \\
N \\
\text { N } \\
\text { ஸे }\end{array}$ & $\begin{array}{l}\text { M } \\
\text { No } \\
\text { ON }\end{array}$ & $\begin{array}{l}\dot{J}_{1} \\
\text { U } \\
\text { d }\end{array}$ & $\begin{array}{l}0 \\
N \\
\text { D } \\
\text { ஸ }\end{array}$ & $\begin{array}{l}\hat{N} \\
\text { D } \\
\text { त }\end{array}$ & $\begin{array}{l}\infty \\
\text { N } \\
\text { d } \\
\text { o }\end{array}$ & $\begin{array}{l}0 \\
N \\
\text { O } \\
\text { డ }\end{array}$ & $\begin{array}{l}\circ \\
\text { N } \\
\text { O } \\
\text { ஸे }\end{array}$ & $\begin{array}{l}\bar{F} \\
\text { N } \\
\text { ¿ }\end{array}$ & $\begin{array}{l}\text { N } \\
\text { No } \\
\text { ரू }\end{array}$ & $\begin{array}{l}\text { m } \\
\text { No } \\
\text { o }\end{array}$ & 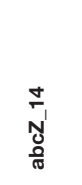 & $\begin{array}{l}\frac{L}{1} \\
\text { No } \\
\frac{0}{\sigma}\end{array}$ & $\begin{array}{l}0 \\
\text { N } \\
\text { চ }\end{array}$ & $\begin{array}{l}\text { N } \\
\text { No } \\
\text { ¿ }\end{array}$ & 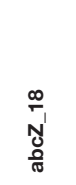 & $\begin{array}{l}\text { Dे } \\
\text { No } \\
\frac{0}{\pi}\end{array}$ & $\begin{array}{l}\text { N } \\
\text { No } \\
\text { ठn }\end{array}$ \\
\hline abcz-F_1574363 & & 98.53 & 98.45 & 98.23 & 96.69 & 97.35 & 96.03 & 88.3 & 96.69 & 96.8 & 83.89 & 95.81 & 97.68 & 96.8 & 96.58 & 97.68 & 98.01 & 83.66 & 96.91 & 88.08 & 98.01 & 96.47 \\
\hline abcZ-R_1574363 & 98.53 & & 98.53 & 98.3 & 96.72 & 97.4 & 96.27 & 88.12 & 96.72 & 96.83 & 84.5 & 96.04 & 97.74 & 96.83 & 96.61 & 97.74 & 98.08 & 84.28 & 96.95 & 87.9 & 98.08 & 96.49 \\
\hline abcZ_5 & 98.45 & 98.53 & & 96.69 & 95.58 & 96.03 & 95.81 & 87.86 & 95.36 & 95.81 & 83.22 & 95.58 & 96.47 & 95.58 & 96.25 & 96.47 & 96.47 & 83 & 95.81 & 87.64 & 96.47 & 95.36 \\
\hline abcz_-1 & 98.23 & 98.3 & 96.69 & & 97.57 & 98.9 & 96.47 & 88.96 & 97.79 & 97.57 & 84.55 & 96.25 & 98.68 & 97.79 & 97.13 & 99.12 & 99.34 & 84.33 & 97.79 & 88.74 & 99.78 & 97.79 \\
\hline abcz_2 & 96.69 & 96.72 & 95.58 & 97.57 & & 96.47 & 96.69 & 90.29 & 99.78 & 96.91 & 84.11 & 96.47 & 97.57 & 98.01 & 96.91 & 97.57 & 97.35 & 83.89 & 97.13 & 90.07 & 97.35 & 99.78 \\
\hline abcZ_3 & 97.35 & 97.4 & 96.03 & 98.9 & 96.47 & & 95.81 & 87.86 & 96.69 & 96.47 & 83.89 & 95.58 & 98.01 & 97.13 & 96.03 & 98.45 & 98.68 & 83.66 & 96.69 & 87.64 & 98.68 & 96.69 \\
\hline abcz_4 & 96.03 & 96.27 & 95.81 & 96.47 & 96.69 & 95.81 & & 88.96 & 96.47 & 94.92 & 84.33 & 99.78 & 96.03 & 97.35 & 97.13 & 96.47 & 95.81 & 84.33 & 95.14 & 88.74 & 96.25 & 96.91 \\
\hline abcz_6 & 88.3 & 88.12 & 87.86 & 88.96 & 90.29 & 87.86 & 88.96 & & 90.07 & 87.86 & 91.39 & 88.96 & 88.52 & 89.4 & 88.3 & 88.96 & 88.3 & 91.17 & 88.3 & 99.78 & 88.74 & 90.51 \\
\hline abcZ_7 & 96.69 & 96.72 & 95.36 & 97.79 & 99.78 & 96.69 & 96.47 & 90.07 & & 97.13 & 84.33 & 96.25 & 97.79 & 98.23 & 96.69 & 97.79 & 97.57 & 84.11 & 97.35 & 89.85 & 97.57 & 99.56 \\
\hline abcz_8 & 96.8 & 96.83 & 95.81 & 97.57 & 96.91 & 96.47 & 94.92 & 87.86 & 97.13 & & 84.77 & 94.7 & 97.13 & 97.13 & 97.35 & 97.13 & 97.35 & 84.55 & 99.34 & 87.64 & 97.35 & 96.69 \\
\hline abcz_9 & 83.89 & 84.5 & 83.22 & 84.55 & 84.11 & 83.89 & 84.33 & 91.39 & 84.33 & 84.77 & & 84.33 & 84.33 & 84.55 & 83.22 & 84.11 & 84.11 & 99.78 & 84.77 & 91.39 & 84.33 & 84.11 \\
\hline abcZ_10 & 95.81 & 96.04 & 95.58 & 96.25 & 96.47 & 95.58 & 99.78 & 88.96 & 96.25 & 94.7 & 84.33 & & 95.81 & 97.13 & 96.91 & 96.25 & 95.58 & 84.33 & 94.92 & 88.74 & 96.03 & 96.69 \\
\hline abcZ_11 & 97.68 & 97.74 & 96.47 & 98.68 & 97.57 & 98.01 & 96.03 & 88.52 & 97.79 & 97.13 & 84.33 & 95.81 & & 98.23 & 96.25 & 99.56 & 98.45 & 84.11 & 96.91 & 88.3 & 98.45 & 97.35 \\
\hline abcZ_12 & 96.8 & 96.83 & 95.58 & 97.79 & 98.01 & 97.13 & 97.35 & 89.4 & 98.23 & 97.13 & 84.55 & 97.13 & 98.23 & & 97.13 & 98.23 & 97.57 & 84.33 & 96.91 & 89.18 & 97.57 & 97.79 \\
\hline abcZ_13 & 96.58 & 96.61 & 96.25 & 97.13 & 96.91 & 96.03 & 97.13 & 88.3 & 96.69 & 97.35 & 83.22 & 96.91 & 96.25 & 97.13 & & 96.69 & 96.47 & 83 & 96.69 & 88.08 & 96.91 & 97.13 \\
\hline abcZ_14 & 97.68 & 97.74 & 96.47 & 99.12 & 97.57 & 98.45 & 96.47 & 88.96 & 97.79 & 97.13 & 84.11 & 96.25 & 99.56 & 98.23 & 96.69 & & 98.45 & 83.89 & 96.91 & 88.74 & 98.9 & 97.79 \\
\hline abcZ_15 & 98.01 & 98.08 & 96.47 & 99.34 & 97.35 & 98.68 & 95.81 & 88.3 & 97.57 & 97.35 & 84.11 & 95.58 & 98.45 & 97.57 & 96.47 & 98.45 & & 83.89 & 97.57 & 88.08 & 99.12 & 97.13 \\
\hline abcZ_16 & 83.66 & 84.28 & 83 & 84.33 & 83.89 & 83.66 & 84.33 & 91.17 & 84.11 & 84.55 & 99.78 & 84.33 & 84.11 & 84.33 & 83 & 83.89 & 83.89 & & 84.55 & 91.17 & 84.11 & 83.89 \\
\hline abcZ_17 & 96.91 & 96.95 & 95.81 & 97.79 & 97.13 & 96.69 & 95.14 & 88.3 & 97.35 & 99.34 & 84.77 & 94.92 & 96.91 & 96.91 & 96.69 & 96.91 & 97.57 & 84.55 & & 88.08 & 97.57 & 96.91 \\
\hline abcZ_18 & 88.08 & 87.9 & 87.64 & 88.74 & 90.07 & 87.64 & 88.74 & 99.78 & 89.85 & 87.64 & 91.39 & 88.74 & 88.3 & 89.18 & 88.08 & 88.74 & 88.08 & 91.17 & 88.08 & & 88.52 & 90.29 \\
\hline abcZ_19 & 98.01 & 98.08 & 96.47 & 99.78 & 97.35 & 98.68 & 96.25 & 88.74 & 97.57 & 97.35 & 84.33 & 96.03 & 98.45 & 97.57 & 96.91 & 98.9 & 99.12 & 84.11 & 97.57 & 88.52 & & 97.57 \\
\hline abcZ_20 & 96.47 & 96.49 & 95.36 & 97.79 & 99.78 & 96.69 & 96.91 & 90.51 & 99.56 & 96.69 & 84.11 & 96.69 & 97.35 & 97.79 & 97.13 & 97.79 & 97.13 & 83.89 & 96.91 & 90.29 & 97.57 & \\
\hline abcZ_21 & 88.08 & 87.9 & 87.64 & 88.74 & 90.07 & 87.64 & 88.74 & 99.78 & 89.85 & 87.64 & 91.17 & 88.74 & 88.3 & 89.18 & 88.08 & 88.74 & 88.08 & 90.95 & 88.08 & 99.56 & 88.52 & 90.29 \\
\hline
\end{tabular}

The 453 nucleotides composing the nucleotide sequence of the abcZ allele sequenced from the specimen no. 1574363 (abcZ-F_1574363 and abcZ-R_1574363) range between $98.45 \%$ with abcZ_5 and $83.66 \%$ with abcZ_16. This table was performed by using Geneious 10.2.3 (Biomatters).

The degree of allele similarity is expressed by a Blue scale scheme code, with the most divergent allelles being displayed in dark blue and the most similar in light blue. 
TABLE 2 | Among the 70 sequence types (STs) of Kingella kingae that are documented in the multilocus sequence database (MLST) of the Institut Pasteur database (http://bigsdb.pasteur.fr/perl/bigsdb/bigsdb.pl?db=pubmlst_kingella_ seqdef_public\&page=downloadProfiles\&scheme_id=1), no. 1574363 shares four alleles, namely, adk-2, cpn60-2, gdh/zwf-13, and recA-2, with ST-26, which belongs to the ST complex (STc)-25; ST-26 is therefore the closest ST to the causative strains no. 1574363.

\begin{tabular}{lcccccccc}
\hline Reference & STc & ST & $\boldsymbol{a b c Z}$ & $\boldsymbol{a d k}$ & $\boldsymbol{a r o E}$ & $\boldsymbol{c p n} 60$ & $\boldsymbol{g d h / z w \boldsymbol { f }}$ & $\boldsymbol{r e c A}$ \\
\hline No. 1574363 & NA & NA & NA & 2 & 2 & 2 & 13 & 2 \\
ST-26 & 25 & 26 & 7 & 2 & 6 & 2 & 13 & 2 \\
\hline
\end{tabular}

NA indicates data not available.

The detection of $K$. kingae is currently improved by sensitive culture methods such as Bactec/Alert vials, and above all by specific qPCR assays $(2,7)$. However, these diagnostic methods are costly and not yet available in developing countries in which diagnostic resources such as blood culture or molecular assays are scarce, and hence the recognition of K. kingae as a possible cause of acute septic arthritis in pediatrics is particularly challenging. In low-income, high-burden settings of tuberculosis, antibiotics with appropriate coverage against $S$. aureus and classical pyogenic bacteria may be frequently administered without any cultures and in the case of non-response to antibiotic treatment, antituberculous drugs may be given empirically for several weeks or months.

Although the child presented with an arthritis caused by K. kingae 4 days after arrival in Southeastern France, we highlight that $K$. kingae infection usually develop in several days to weeks following oropharyngeal $K$. kingae carriage and viral infections (18). Moreover, MLST analysis of invasive K. kingae strains from Southeastern France in 2016 demonstrated that strains causing osteoarticular infections belonged to ST- 6 and ST-25 in the large majority of cases (14). Taken together with the novel K. kingae STc herein described, these findings are consistent with the fact that the child acquired causative K. kingae strains in Cameroon.

Notably, in an unpublished pilot study, $K$. kingae has been identified in the oropharynx of young children from Western Africa. This study was carried out at the Donka University Hospital in Conakry, Guinea, from 2012 to 2013 (Ceroni and Lamah, unpublished data). To define the prevalence rate of oropharyngeal $K$. kingae carriage, 45 healthy children aged from 6 to 48 months were enrolled in this study. Children admitted for either elective surgery or attending the orthopedic outpatient clinic or visiting the emergency department for non-infectious disease were included, whereas those presenting an invasive infectious disease, or administration of antimicrobial drugs the two preceding months were excluded. Recent travel abroad was not reported in any child. Oropharyngeal specimens were obtained by rubbing a cotton swab on the child's tonsils, which were subsequently tested by molecular assays described earlier (13). Three children tested positive for $K$. kingae, thus indicating a prevalence rate of $6.7 \%$, which is roughly similar to that observed in Europe (4). Despite the small size of this pilot study, these preliminary results provide evidence that $K$. kingae is circulating in Western Africa as well, and as a result, $K$. kingae might be considered as a potential pathogen responsible for septic
TABLE 3 | Multilocus sequence typing (MLST) scheme of Kingella kingae shows the combination of the six alleles used to define the sequence types (STs) and sequence-type complexes (STcs) of $K$. kingae.

\begin{tabular}{|c|c|c|c|c|c|c|c|}
\hline STc & ST & $a b c Z$ & $a d k$ & aroE & cpn60 & $g d h / z w f$ & recA \\
\hline 1 & 1 & 1 & 1 & 1 & 1 & 1 & 1 \\
\hline 1 & 2 & 1 & 1 & 1 & 1 & 1 & 3 \\
\hline 3 & 3 & 14 & 9 & 14 & 1 & 7 & 4 \\
\hline NA & 4 & 3 & 3 & 9 & 3 & 7 & 3 \\
\hline NA & 5 & 4 & 2 & 9 & 3 & 7 & 3 \\
\hline 6 & 6 & 5 & 2 & 4 & 5 & 5 & 1 \\
\hline 6 & 7 & 5 & 2 & 13 & 5 & 5 & 1 \\
\hline NA & 8 & 11 & 2 & 3 & 7 & 7 & 2 \\
\hline NA & 9 & 11 & 2 & 4 & 3 & 4 & 3 \\
\hline NA & 10 & 1 & 8 & 3 & 6 & 1 & 3 \\
\hline 11 & 11 & 13 & 2 & 4 & 2 & 8 & 6 \\
\hline 11 & 12 & 15 & 2 & 4 & 2 & 8 & 6 \\
\hline NA & 13 & 3 & 3 & 3 & 3 & 10 & 4 \\
\hline 14 & 14 & 3 & 3 & 3 & 3 & 3 & 3 \\
\hline 14 & 15 & 3 & 3 & 3 & 3 & 12 & 3 \\
\hline 14 & 16 & 3 & 3 & 12 & 3 & 3 & 3 \\
\hline 14 & 17 & 3 & 2 & 3 & 3 & 3 & 3 \\
\hline 14 & 18 & 8 & 3 & 3 & 3 & 3 & 3 \\
\hline NA & 19 & 4 & 4 & 4 & 4 & 1 & 3 \\
\hline NA & 20 & 4 & 2 & 3 & 4 & 1 & 3 \\
\hline 23 & 21 & 10 & 2 & 2 & 2 & 2 & 2 \\
\hline 23 & 22 & 4 & 2 & 2 & 2 & 2 & 2 \\
\hline 23 & 23 & 2 & 2 & 2 & 2 & 2 & 2 \\
\hline 23 & 24 & 2 & 2 & 8 & 2 & 2 & 2 \\
\hline 25 & 25 & 7 & 2 & 6 & 2 & 2 & 2 \\
\hline 25 & 26 & 7 & 2 & 6 & 2 & 13 & 2 \\
\hline NA & 27 & 12 & 6 & 10 & 3 & 9 & 2 \\
\hline 29 & 28 & 9 & 2 & 7 & 3 & 4 & 3 \\
\hline 29 & 29 & 9 & 2 & 4 & 3 & 4 & 3 \\
\hline NA & 30 & 16 & 10 & 7 & 3 & 4 & 3 \\
\hline NA & 31 & 6 & 1 & 4 & 3 & 1 & 5 \\
\hline 32 & 32 & 6 & 5 & 5 & 3 & 6 & 5 \\
\hline NA & 33 & 6 & 7 & 11 & 3 & 11 & 5 \\
\hline NA & 34 & 6 & 7 & 11 & 3 & 2 & 5 \\
\hline 35 & 35 & 1 & 8 & 15 & 8 & 1 & 3 \\
\hline NA & 36 & 1 & 11 & 15 & 8 & 1 & 3 \\
\hline NA & 37 & 3 & 3 & 3 & 3 & 2 & 3 \\
\hline NA & 38 & 6 & 7 & 11 & 3 & 2 & 2 \\
\hline 3 & 39 & 14 & 9 & 14 & 1 & 7 & 10 \\
\hline NA & 40 & 9 & 2 & 7 & 10 & 4 & 3 \\
\hline 14 & 41 & 3 & 3 & 9 & 3 & 3 & 3 \\
\hline 14 & 42 & 3 & 3 & 3 & 3 & 14 & 3 \\
\hline NA & 43 & 3 & 2 & 3 & 3 & 15 & 11 \\
\hline
\end{tabular}


TABLE 3 | Continued

\begin{tabular}{|c|c|c|c|c|c|c|c|}
\hline STC & ST & $a b c Z$ & adk & aroE & cpn60 & $g d h / z w f$ & recA \\
\hline 23 & 44 & 4 & 2 & 2 & 2 & 2 & 7 \\
\hline 6 & 45 & 5 & 2 & 4 & 5 & 5 & 9 \\
\hline 6 & 46 & 5 & 2 & 6 & 5 & 5 & 1 \\
\hline NA & 47 & 6 & 7 & 11 & 3 & 17 & 12 \\
\hline NA & 48 & 1 & 1 & 17 & 2 & 16 & 2 \\
\hline NA & 49 & 1 & 1 & 17 & 9 & 16 & 2 \\
\hline NA & 50 & 17 & 1 & 1 & 11 & 1 & 8 \\
\hline NA & 51 & 4 & 6 & 9 & 4 & 1 & 3 \\
\hline 35 & 52 & 1 & 8 & 3 & 8 & 1 & 3 \\
\hline NA & 53 & 18 & 2 & 4 & 2 & 9 & 3 \\
\hline NA & 54 & 3 & 2 & 3 & 3 & 1 & 11 \\
\hline 23 & 55 & 19 & 2 & 2 & 2 & 2 & 2 \\
\hline 23 & 56 & 20 & 2 & 6 & 2 & 2 & 2 \\
\hline 14 & 57 & 3 & 3 & 3 & 3 & 18 & 3 \\
\hline 35 & 58 & 1 & 8 & 15 & 8 & 19 & 3 \\
\hline 6 & 59 & 5 & 2 & 18 & 5 & 5 & 1 \\
\hline 14 & 60 & 8 & 3 & 3 & 3 & 3 & 13 \\
\hline 6 & 61 & 5 & 2 & 4 & 5 & 20 & 1 \\
\hline 23 & 62 & 2 & 2 & 6 & 2 & 2 & 2 \\
\hline NA & 63 & 14 & 2 & 19 & 1 & 7 & 10 \\
\hline NA & 64 & 3 & 2 & 16 & 3 & 3 & 3 \\
\hline NA & 65 & 21 & 7 & 11 & 3 & 17 & 5 \\
\hline 32 & 66 & 6 & 5 & 10 & 3 & 6 & 5 \\
\hline NA & 67 & 5 & 2 & 3 & 2 & 2 & 2 \\
\hline NA & 68 & 5 & 2 & 6 & 11 & 9 & 1 \\
\hline NA & 69 & 1 & 2 & 6 & 2 & 1 & 14 \\
\hline 23 & 70 & 2 & 2 & 20 & 2 & 2 & 2 \\
\hline NA & NA & NA & 2 & 2 & 2 & 13 & 2 \\
\hline
\end{tabular}

NA indicates data not yet available.

In the present case, MLST sequencing data from the joint fluid specimen no. 1574363 indicated that the K. kingae causative strains shared four alleles with ST-26/STC-

25, namely, adk-2, cpn60-2, gdh/zwf-13, and recA-2. Therefore, ST-26/STc-25 is the closest ST with the K. kingae strains that were identified in the synovial fluid no. 1574363 (boxes designed on blue background in the bottom row of the table). Please note that among the 70 STs identified, $31 \mathrm{~K}$. kingae isolates have not yet an STC determined (boxes designed on orange background).

arthritis in young children living in this geographical area. Early microbiologically proven diagnosis of $K$. kingae infection would enable to provide appropriate antibiotic therapy by amoxicillin, or amoxicillin-clavulanate, and to drastically reduce the total duration of treatment to a few days or weeks $(2,6)$. This would also make it possible to avoid the administration of potentially harmful antituberculous regimens.

\section{REFERENCES}

1. Yagupsky P. Kingella kingae: from medical rarity to an emerging paediatric pathogen. Lancet Infect Dis (2004) 4:358-67. doi:10.1016/S14733099(04)01046-1

\section{CONCLUDING REMARKS}

This case report demonstrates that $K$. kingae might be considered as a potential cause of acute septic arthritis in children living in sub-Saharan Africa. Together with the evidence of $K$. kingae carriage among healthy children from Western Africa, these findings suggest that $K$. kingae might contribute to an underestimated burden of septic arthritis in this geographical area. Moreover, MLST analysis disclosed the first K. kingae STc in Africa that is a novel STc close to ST-26. Further prospective studies to specify the prevalence of $K$. kingae infection and carriage in sub-Saharan Africa are required to better help guiding rational diagnostic and therapeutic strategies.

\section{CONSENT FOR PUBLICATION}

The written consent for publication was obtained from the parents' child.

\section{ETHICS STATEMENT}

The study was approved by the Ethics committee of the IHU Mediterranee-Infection under reference number 2016-024.

\section{AUTHOR CONTRIBUTIONS}

All the authors provided a substantial contribution to the conception and design of the work, and acquisition, analysis, and interpretation of data for the work. NEH and DC drafted the initial version of the manuscript, and all the authors revised it critically for important intellectual content. All the authors approved the present version to be published.

\section{ACKNOWLEDGMENTS}

The authors are grateful to the patient's parents, as well as children and their family from Conakry, Guinea, for participating to this work.

\section{FUNDING}

This work was supported by the Mediterranee Infection foundation (http://www.mediterranee-infection.com/article.php? larub $=126 \&$ titer=la-fondation-recrute) through a $\mathrm{PhD}$ grant awarded to NEH from 2015 to 2017.

\section{SUPPLEMENTARY MATERIAL}

The Supplementary Material for this article can be found online at http://www.frontiersin.org/article/10.3389/fped.2017.00230/ full\#supplementary-material.

2. Yagupsky P, Porsch E, St Geme JW. Kingella kingae: an emerging pathogen in young children. Pediatrics (2011) 127:557-65. doi:10.1542/peds.2010-1867

3. Amit U, Dagan R, Yagupsky P. Prevalence of pharyngeal carriage of Kingella kingae in young children and risk factors for colonization. Pediatr Infect Dis $J$ (2013) 32:191-3. doi:10.1097/INF.0b013e3182755779 
4. Ceroni D, Llana RA, Kherad O, Dubois-Ferriere V, Lascombes P, Renzi G, et al. Comparing the oropharyngeal colonization density of Kingella kingae between asymptomatic carriers and children with invasive osteoarticular infections. Pediatr Infect Dis J (2013) 32:412-4. doi:10.1097/INF. 0b013e3182846e8f

5. Olijve L, Podmore R, Anderson T, Walls T. High rate of oropharyngeal Kingella kingae carriage in New Zealand children. J Paediatr Child Health (2016) 52:1081-5. doi:10.1111/jpc.13287

6. Yagupsky P. Kingella kingae: carriage, transmission, and disease. Clin Microbiol Rev (2015) 28:54-79. doi:10.1128/CMR.00028-14

7. El Houmami N, Bzdrenga J, Durand GA, Minodier P, Seligmann H, Prudent E, et al. Molecular tests that target the RTX locus do not distinguish between Kingella kingae and the recently described Kingella negevensis species. JClin Microbiol (2017) 55:3113-22. doi:10.1128/ JCM.00736-17

8. Basmaci R, Bidet P, Yagupsky P, Muñoz-Almagro C, Balashova NV, Doit C, et al. Major intercontinentally distributed sequence types of Kingella kingae and development of a rapid molecular typing tool. J Clin Microbiol (2014) 52:3890-7. doi:10.1128/JCM.01609-14

9. Kuzumoto K, Kubota N, Saito Y, Fujioka F, Yumoto K, Hidaka R, et al. A case of osteomyelitis due to Kingella kingae. Kansenshogaku Zasshi (2013) 87:207-10. doi:10.11150/kansenshogakuzasshi.87.207

10. Osei L, El Houmami N, Minodier P, Sika A, Basset T, Seligmann H, et al. Paediatric bone and joint infections in French Guiana: a 6 year retrospective review. J Trop Pediatr (2017) 63:380-8. doi:10.1093/tropej/fmw102

11. Levy PY, Fournier PE, Fenollar F, Raoult D. Systematic PCR detection in culture-negative osteoarticular infections. Am J Med (2013) 126:1143.e25-33. doi:10.1016/j.amjmed.2013.04.027

12. Bruijnesteijn van Coppenraet ES, Lindeboom JA, Prins JM, Peeters MF, Claas EC, Kuijper EJ. Real-time PCR assay using fine-needle aspirates and tissue biopsy specimens for rapid diagnosis of mycobacterial lymphadenitis in children. J Clin Microbiol (2004) 42:2644-50. doi:10.1128/JCM.42. 6.2644-2650.2004
13. Cherkaoui A, Ceroni D, Emonet S, Lefevre Y, Schrenzel J. Molecular diagnosis of Kingella kingae osteoarticular infections by specific real-time PCR assay. J Med Microbiol (2009) 58:65-8. doi:10.1099/jmm.0.47707-0

14. El Houmami N, Bzdrenga J, Pons JC, Minodier P, Durand GA, Oubraham A, et al. A modified multilocus sequence typing protocol to genotype Kingella kingae from oropharyngeal swabs without bacterial isolation. BMC Microbiol (2017) 17:200. doi:10.1186/s12866-017-1104-5

15. Basmaci R, Yagupsky P, Ilharreborde B, Guyot K, Porat N, Chomton M, et al. Multilocus sequence typing and $r t x A$ toxin gene sequencing analysis of Kingella kingae isolates demonstrates genetic diversity and international clones. PLoS One (2012) 7:e38078. doi:10.1371/journal.pone.0038078

16. Stoesser N, Pocock J, Moore CE, Soeng S, Hor P, Sar P, et al. The epidemiology of pediatric bone and joint infections in Cambodia, 2007-11. J Trop Pediatr (2013) 59:36-42. doi:10.1093/tropej/fms044

17. Ceroni D, Cherkaoui A, Combescure C, François P, Kaelin A, Schrenzel J. Differentiating osteoarticular infections caused by Kingella kingae from those due to typical pathogens in young children. Pediatr Infect Dis J (2011) 30:906-9. doi:10.1097/INF.0b013e31821c3aee

18. El Houmami N, Minodier P, Dubourg G, Mirand A, Jouve JL, Basmaci R, et al. Patterns of Kingella kingae disease outbreaks. Pediatr Infect Dis J (2016) 35:340-6. doi:10.1097/INF.0000000000001010

Conflict of Interest Statement: The authors declare that the research was conducted in the absence of any commercial or financial relationships that could be construed as a potential conflict of interest.

Copyright (c) 2017 El Houmami, Ceroni, Codjo Seignon, Pons, Lambert, Durand, Minodier, Lamah, Bidet, Schrenzel, Raoult and Fournier. This is an open-access article distributed under the terms of the Creative Commons Attribution License (CC $B Y)$. The use, distribution or reproduction in other forums is permitted, provided the original author(s) or licensor are credited and that the original publication in this journal is cited, in accordance with accepted academic practice. No use, distribution or reproduction is permitted which does not comply with these terms. 